\title{
Comments on "Socio-geographical factors in vulnerability to dengue in Thai villages: a spatial regression analysis" by Tipayamongkholgul and Lisakulruk
}

Dear Editor,

We compliment Tipayamongkholgul and Lisakulruk for their spatial regression analysis of different local socio-geographic predictors of dengue infection in the Thai province of Prachuap Khiri Kan, published in Geospatial Health, issue 5.2 (2011). However, for such a comprehensive analysis, we feel that it is essential that full details about the local vectors and the casual and formal dress worn by the villagers during the day-time are discussed and evaluated.

The biology of the vector Aedes albopictus and Ae. aegypti, responsible for dengue fever and chikungunya fever, is intriguing. Ae. albopictus is a very aggressive day-time biter, with peaks generally occurring during early morning and late afternoon. It is a container-inhabiting species laying its eggs in any water-containing receptacle in urban, suburban, rural and forested areas (Novak, 1992). Furthermore, Ae. aegypti is also any early-morning or late-afternoon biter, but will also bite at night if there is sufficient artificial light. Importantly, Ae. aegypti is particularly fond of ankles.

Conventionally, the casual and/or formal dress worn by every individual in this part of the world varies during the day. Any dress that hides the lower extremities during day time would offer protection against bites by mosquitoes of the genus Aedes. However, every inadvertent day-time exposure of the lower extremities, while on leisure or formal employment, would be associated with a higher probability of being bitten and infected by a mosquito carrying the dengue virus.

A prospective, long-term study measuring the ecological impact of urbanization of dengue endemicity should also have addressed the description of anti-vector measures being adapted during the interim phase. Generally, the anti-mosquito measures are practiced by individuals during the night when repellents, insecticides or mosquito nets are used. Unfortunately, the nocturnal use of insect repellents, the wearing of permethrin-impregnated clothing, sleeping under permethrin-impregnated bed nets, and staying in accommodations with screened or air-conditioned rooms (Centers for Disease Control, 2009) would not completely protect a villager, as he or she would be exposed to the potential risk of bites by Aedes mosquitoes during day-time activities.

\section{References}

Centers for Disease Control and Prevention, 2009. CDC health information for international travel 2010. Atlanta: US Department of Health and Human Services, Public Health Service.

Novak R, 1992. The Asian tiger mosquito, Aedes albopictus. Wing Beats 3, 5 .

Tipayamongkholgul M, Lisakulruk S, 2011. Socio-geographical factors in vulnerability to dengue in Thai villages: a spatial regression analysis. Geospat Health 5, 191-198.

Subhash C. Arya and Nirmala Agarwal

Department of Clinical Microbiology and Infection Control Sant Parmanand Hospital 18 Alipore Road Delhi-110054, India E-mail:subhashbhapaji@mail.com 